Literature Reviews

\title{
Book Review: Quantum Field Theory
}

\author{
Christina Pospisil \\ Department of Physics, Mathematics, Computer Science, University of Massachusetts Boston, USA
}

The textbook presents a summary about Quantum Field Theory (QFT) with mathematical details. A wide range of main concepts and additional topics in quantum field theory is displayed in the academic textbook to scientists. Field theory describes and represents phenomena physically and mathematically through forces acting and spreading as field in space. Quantum field theory is constructed and introduced from the basic principles of special relativity, mode decomposition, the Kaellen Lehmann Spectral Representation, et al. and perturbation theory. Perturbation Theory presents a tool for calculating from a solution of a given condition of a physical system the approximate solutions for small perturbed conditions of the original case. Further important introduced main terms are the concept of the harmonic oscillator, Noether's Theorem, the concept of relativistical quantum mechanics, spontaneous symmetry breaking regarding Lagrangian symmetries, which are not found at the ground state level and the nuclean pion interaction called the Linear Sigma Model. The book continous with path integrals, path integral formalism, the Schwinger-Dyson Equations and Grassman variables. Moreover, main parts of the book are a physical and mathematical description of Quantum Field Theories, which take effect and are effective with the fields from matter (these are the Gauge theories) and mathematical concepts behind lie groups and lie algebras, Ghost Fields, the FadeevPopov Quantization, Feynman Rule, renormalizability and the renormalization group as a tool for a more detailed analysis of quantum field phenomena. Quantum Field Theory is also a book, that includes information beside the classical description; that means e.g., anomalies caused by quantum effects are summarized in a separate chapter. Paramount experimental tools are observables, which can be translated into theoretical physics concepts also via tree amplitudes. The final chapters of the book include an extension of the classical perturbation theory, worldline formalism, an analysis of correspondings regarding the concept of trees and loops in quantum field theory and lattice field theory. In addition the book includes a section about further reading and each chapter includes exercises. Quantum Field Theory can serve for self-study and reference guide for the reader.

The textbook Quantum Field Theory, author: Francois Gelis, edition one, publication date: June 2019 is published by the Cambridge University Press.

ISBN: 9781108691550

CUP-weblink: https://www.cambridge.org/core/books/quantum-field-theory/5484B936BB6323A0991E334AE9A02A49 